\title{
Stability of the neutron-proton-electron matter under strong magnetic fields: The covariant Vlasov approach
}

\author{
Sidney Avancini, ${ }^{1,2}$ B. P. Bertolino, ${ }^{2}$ Aziz Rabhi, ${ }^{1,3}$ Jianjun Fang, ${ }^{1,4}$ Helena Pais, ${ }^{1}$ and Constança Providência ${ }^{1}$ \\ ${ }^{1}$ CFisUC, Department of Physics, University of Coimbra, 3004-516 Coimbra, Portugal \\ ${ }^{2}$ Departamento de Física, Universidade Federal de Santa Catarina, Florianópolis, SC, CP. 476, CEP 88.040-900, Brazil \\ ${ }^{3}$ University of Carthage, Avenue de la République BP 77 -1054 Amilcar, Tunisia \\ ${ }^{4}$ School of Physics and Physical Engineering, Qufu Normal University, 273165 Qufu, China
}

(Received 25 June 2018; published 29 August 2018)

\begin{abstract}
The neutron-proton-electron matter under a strong magnetic field is studied in the context of the covariant Vlasov approach. We use a Walecka-type hadronic model and the dispersion relations for the longitudinal and transverse modes are obtained. The instability regions for longitudinal and transverse modes are also studied. The crust-core transition of a magnetized neutron star is discussed.
\end{abstract}

DOI: 10.1103/PhysRevC.98.025805

\section{INTRODUCTION}

Neutron stars are astrophysical objects with extreme properties and very high densities in their interior, which could be as high as one order of magnitude larger than nuclear matter at saturation densities, very intense surface magnetic fields, which can reach $10^{15} \mathrm{G}$ in the case of magnetars [1], and very large isospin asymmetries. These properties transform neutron stars into effective laboratories for understanding the strong force. Objects such as soft $\gamma$-ray repeaters and anomalous $\mathrm{x}$-ray pulsars have been identified as magnetars carrying very strong surface magnetic fields [2]. They have a slow rotation period, $\approx 1-12 \mathrm{~s}$, and this has been interpreted as a signature of the existence of an amorphous and heterogeneous layer, with a high electrical resistivity, close to the crust-core transition, which causes the decay of the magnetic field [3].

The inner crust of neutron stars is characterized by subsaturation nuclear densities and it is formed by a lattice of neutron-rich clusters imbedded in a neutron and electron gas background. In fact, at subsaturation densities, nuclear matter is characterized by a liquid-gas phase transition. As a consequence of long-range Coulomb repulsion and short-range nuclear attraction this transition originates the formation of clusterized matter that, depending on the density, may have exotic geometries, and which are known as nuclear pasta [4]. At the bottom of the inner crust, where these clusters occur, the neutron star core sets in. Understanding how the magnetic field may affect the localization of the core onset has been the objective of recent works [5-7], where the crust-core transition in the presence of strong magnetic fields was studied within the calculation of the dynamical spinodal section [8]. From the linearization of the Vlasov equation for the distribution function of protons, neutrons, and electrons, the dispersion relation for the propagation of longitudinal models along the magnetic field was calculated. The spinodal section was identified with the surface in phase space where the density mode frequencies are zero. The main conclusions of those works were that the magnetic field shifts the onset of the outer core to larger densities, the crust-core transition is a region of finite density thickness, the density location of this transition region formed by alternating stable and unstable layers was quite sensitive to the slope of the symmetry energy, and temperatures of the order of $\approx 0.5-1 \mathrm{MeV}$ might wash out most of these effects.

One of the main objectives of the present work is to obtain the dispersion relations for neutron-proton-electron (npe) matter subjected to a strong external magnetic field within the formalism of the covariant Wigner function, which allows us to calculate the propagation of density modes in an arbitrary direction with respect to direction of the magnetic field. The dispersion relations will be applied to the calculation of the transverse and longitudinal collective modes, both stable and unstable. The unstable modes determine the dynamical spinodal zones, which set boundaries for the inhomogeneous region of the magnetar crust. This formalism is of particular interest because it may be easily generalized in order to calculate the electrical and thermal conductivity in the magnetized matter, which is of fundamental importance for the study of the cooling of magnetars.

\section{FORMALISM}

The Lagrangian density using natural units, i.e., taking $c=\hbar=1$, can be written as

$$
\mathcal{L}=\sum_{j=p, n, e} \mathcal{L}_{j}+\mathcal{L}_{\sigma}+\mathcal{L}_{\omega}+\mathcal{L}_{\rho}+\mathcal{L}_{\omega \rho}+\mathcal{L}_{A}
$$

with

$$
\mathcal{L}_{j}=\bar{\psi}^{(j)}\left[\gamma^{\mu} i D_{\mu}^{(j)}-M_{j}^{*}\right] \psi^{(j)},
$$

where the covariant derivative is defined as, $i D_{\mu}^{(j)}=i \partial_{\mu}-$ $\mathcal{V}_{\mu}^{(j)}$, where $j=(n, p, e)$, stands for the neutron, proton, and 
electron,

$$
\mathcal{V}_{\mu}^{(j)}=\left\{\begin{array}{ll}
g_{v} V_{\mu}+\frac{g_{\rho}}{2} \vec{b}_{\mu}+e A_{\mu}, & j=p \\
g_{v} V_{\mu}-\frac{g_{\rho}}{2} \vec{b}_{\mu}, & j=n \\
-e A_{\mu}, & j=e
\end{array},\right.
$$

$M_{p}^{*}=M_{n}^{*}=M^{*}=M-g_{s} \phi(x), \quad M_{e}^{*}=m_{e} \quad$ and $\quad e=$ $\sqrt{4 \pi / 137}$ is the electromagnetic coupling constant. For the nuclear matter parameters, we will consider the NL3 parametrization, [9], and the FSU parametrization [10]. The meson and photon contributions in Eq. (1) are given by

$$
\begin{aligned}
\mathcal{L}_{\sigma} & =\frac{1}{2}\left(\partial_{\mu} \phi \partial^{\mu} \phi-m_{s}^{2} \phi^{2}-\frac{1}{3} \kappa \phi^{3}-\frac{1}{12} \lambda \phi^{4}\right), \\
\mathcal{L}_{\omega} & =\frac{1}{2}\left(-\frac{1}{2} \Omega_{\mu \nu} \Omega^{\mu \nu}+m_{v}^{2} V_{\mu} V^{\mu}+\frac{1}{12} \xi g_{v}^{4}\left(V_{\mu} V^{\mu}\right)^{2}\right), \\
\mathcal{L}_{\rho} & =\frac{1}{2}\left(-\frac{1}{2} \vec{B}_{\mu \nu} \cdot \vec{B}^{\mu \nu}+m_{\rho}^{2} \vec{b}_{\mu} \cdot \vec{b}^{\mu}\right), \\
\mathcal{L}_{\omega \rho} & =\Lambda_{v} g_{v}^{2} g_{\rho}^{2} V_{\mu} V^{\mu} \vec{b}_{\nu} \cdot \vec{b}^{\nu} \\
\mathcal{L}_{A} & =-\frac{1}{4} F_{\mu \nu} F^{\mu \nu},
\end{aligned}
$$

where $\Omega_{\mu \nu}=\partial_{\mu} V_{\nu}-\partial_{\nu} V_{\mu}, \vec{B}_{\mu \nu}=\partial_{\mu} \vec{b}_{v}-\partial_{\nu} \vec{b}_{\mu}-\Gamma_{\rho}\left(\vec{b}_{\mu} \times\right.$ $\vec{b}_{v}$ ), and $F_{\mu \nu}=\partial_{\mu} A_{\nu}-\partial_{\nu} A_{\mu}$. The parameters $\kappa, \lambda$, and $\xi$ are self-interacting couplings and the $\omega-\rho$ coupling $\Lambda_{v}$ is included to soften the density dependence of the symmetry energy above saturation density. From the Euler-Lagrange equations, one obtains the Dirac equation for the fermion fields:

$$
i \gamma^{\mu} D_{\mu}^{(j)} \psi^{(j)}=M_{j}^{\star} \psi^{(j)},
$$

and its conjugate equation:

$$
\bar{\psi}^{(j)} i D_{\mu}^{\dagger(j)} \gamma^{\mu}=-M_{j}^{\star} \bar{\psi}^{(j)},
$$

where $i D_{\mu}^{\dagger(j)}=i \overleftarrow{\partial}_{\mu}+\mathcal{V}_{\mu}^{(j)}$. In this section, we will discuss how the Vlasov equation for a hadronic system in a strong magnetized medium is obtained from general transport equations. Our formalism is based on the covariant Wigner function under strong magnetic fields. The transport equations using Wigner functions have been developed in the context of quantum electrodynamics [11,12], relativistic heavy ions [13,14], and quantum chromodynamics [15]. The application of the Wigner function for a relativistic electron gas in a strong magnetic field was done in Refs. [16-19]. We will present in this section only the main results related to the transport theory in order to keep this paper minimally self-contained. We will focus on the new technical details that appear when using the covariant Wigner function for the description of npe matter subjected to strong magnetic fields since, in the present context and to our knowledge, this has not yet been done. It will be shown that both the longitudinal and transverse modes, which consist of small oscillations along and perpendicular to the external magnetic field, can be obtained in a systematic way by generalizing magnetized plasma physics techniques. The present formalism is adequate for the study of collective modes and opens the possibility to study thermal and electrical conductivity in npe matter.

\section{A. Covariant Wigner function}

We define the Wigner covariant matrix operator [12] as:

$$
\hat{W}_{4}^{(j)}(x, p)=\int d^{4} y e^{-i p \cdot y} \Phi_{4}^{(j)}(x, y),
$$

where $x$ and $y$ are four-vectors, $j=(n, p, e)$, and

$$
\begin{aligned}
& \Phi_{4}^{(j)}(x, y)_{\alpha \beta} \equiv \bar{\psi}_{\beta}^{(j)}(x) e^{\frac{y}{2} \cdot D^{\dagger(j)}} e^{-\frac{y}{2} \cdot D^{(j)}} \psi_{\alpha}^{(j)}(x) \\
& =\bar{\psi}_{\beta}^{(j)}\left(x_{+}\right) e^{-i y \cdot \int_{-1 / 2}^{1 / 2} d s \mathcal{V}^{(j)}(x+y s)} \psi_{\alpha}^{(j)}\left(x_{-}\right) .
\end{aligned}
$$

In the latter equation, $x_{ \pm}=x \pm \frac{1}{2} y$, and the phase factor contains a line integra, which is to be calculated along a straight line path from $x_{-}$to $x_{+}$, since in this case the gauge invariance of the Wigner function is guaranteed [11]. If one defines the canonical, $\hat{p}_{\mu}$, and kinetic, $\hat{\Pi}_{\mu}$, momentum operators as:

$\hat{p}_{\mu}=\frac{1}{2 i}\left(\overleftarrow{\partial}_{\mu}-\partial_{\mu}\right), \quad \hat{\Pi}_{\mu}=\frac{1}{2 i}\left(D_{\mu}^{\dagger(j)}-D_{\mu}^{(j)}\right)=\hat{p}_{\mu}-\mathcal{V}_{\mu}^{(j)}$

then, from Eqs. (6), (7), the Wigner matrix can be rewritten as:

$$
\hat{W}_{4}^{(j)}(x, p)_{\alpha \beta}=(2 \pi)^{4} \bar{\psi}_{\beta}^{(j)}(x) \delta^{4}[\hat{\Pi}(x)-p] \psi_{\alpha}^{(j)}(x) .
$$

From the Dirac equations, (4), (5), in a rather cumbersome calculation $[11,12]$, it is possible to derive the following equation for $\hat{W}_{4}(x, p)$ :

$$
\left[\gamma^{\mu}\left(\hat{\Pi}_{\mu}+\frac{i \hbar}{2} \mathcal{D}_{\mu}\right)-M^{\star}\left(x-\frac{i \hbar}{2} \partial_{p}\right)\right] \hat{W}_{4}(x, p)=0,
$$

where

$$
\begin{aligned}
& \mathcal{D}_{\mu}=\partial_{x \mu}-\int_{-1 / 2}^{1 / 2} d s \mathcal{F}_{\mu \nu}\left(x+i \hbar s \partial_{p}\right) \partial_{p}^{\nu}, \\
& \hat{\Pi}_{\mu}=p_{\mu}+i \hbar \int_{-1 / 2}^{1 / 2} d s s \mathcal{F}_{\mu \nu}\left(x+i \hbar s \partial_{p}\right) \partial_{p}^{\nu},
\end{aligned}
$$

and $\mathcal{F}_{\mu \nu}^{(j)}=\partial_{x \mu} \mathcal{V}_{\nu}^{(j)}-\partial_{x \nu} \mathcal{V}_{\mu}^{(j)}$. In Eq. (8)

$$
M^{\star}\left(x-\frac{i \hbar}{2} \partial_{p}\right)=\sum_{n=0}^{\infty} \frac{1}{n !}\left(\frac{-i \hbar}{2}\right)^{n} \Delta^{n} M^{\star}(x),
$$

with $\Delta=\partial_{x} \cdot \partial_{p}$, and $\Delta M^{\star}(x)=\left(\frac{\partial}{\partial x_{\mu}} M^{\star}(x)\right) \frac{\partial}{\partial p_{\mu}}$. Note that $\partial_{x \mu} \equiv \partial_{\mu}$. The first designation will be used if necessary to distinguish from the four-gradient with respect to $p$.

In the latter equations, $\hbar$ is explicitly shown, since our aim is to consider approximations of $O(\hbar)$ in order to derive the semiclassical Vlasov equation. From here on, we will omit the particle index, $(j)$, whenever this does not cause confusion. Notice that Eq. (8), when $\hbar \rightarrow 0$, can be written as:

$$
\left[p^{2}-M^{\star}(x)^{2}\right] \hat{W}_{4}(x, p)=0
$$

or

$$
\left\{p^{0^{2}}-\left[\vec{p}^{2}+M^{\star}(x)^{2}\right]\right\} \hat{W}_{4}(x, p)=0 .
$$

This result means that the Wigner operator is on the mass shell, thus, the component of $O\left(\hbar^{0}\right)$ of $\hat{W}_{4}$ can be written as:

$$
\hat{W}_{4}(x, p)=\hat{W}_{4}^{(+)(0)} \delta\left(p_{0}-E_{p}\right)+\hat{W}_{4}^{(-)(0)} \delta\left(p_{0}+E_{p}\right),
$$


where $E_{p}=\sqrt{\vec{p}^{2}+M^{\star}(x)^{2}}$. Here, we will discuss briefly how the Vlasov equation may be obtained from the Wigner operator. First [11], we expand the Wigner $4 \times 4$ matrix operator in terms of the Clifford algebra:

$$
\begin{aligned}
\hat{W}_{4}(x, p)= & \frac{1}{4}\left[F(x, p) I+i \gamma^{5} P(x, p)+\gamma^{\mu} F_{\mu}(x, p) .\right. \\
& \left.+\gamma^{\mu} \gamma^{5} \Omega_{\mu}(x, p)+\frac{1}{2} \sigma^{\mu \nu} S_{\mu \nu}(x, p)\right],
\end{aligned}
$$

where $\sigma^{\mu \nu}=\frac{1}{2}\left(\gamma^{\mu} \gamma^{\nu}-\gamma^{\nu} \gamma^{\mu}\right)$. After substituting the Clifford expansion of $\hat{W}_{4}$ in Eq. (8), we calculate the traces in Dirac space by multiplying the resulting expression by each one of the sixteen Clifford algebra elements. Thus, we obtain 16 complex equations [12]. Next, we consider the energy average of the Wigner function, since the Vlasov equation is usually written in terms of the Cartesian momentum vector, $\vec{p}$, instead of the four-vector $p^{\mu}$. For convenience, we define $\hat{W}_{3}(x, \vec{p})$ as:

$$
\hat{W}_{3}(x, \vec{p})=\int d p_{0} \hat{W}_{4}\left(x, p^{0}, \vec{p}\right) \gamma^{0} .
$$

Of course, one obtains again 16 equations relating the components of the $\hat{W}_{3}$ just defined. It is possible to show [12], after a long and tedious calculation, that at $O(\hbar)$ one obtains the following generalized Vlasov equation:

$$
\begin{aligned}
& \partial_{t} f_{ \pm} \pm \vec{v} \cdot \nabla_{x} f_{ \pm}+(\overrightarrow{\mathcal{E}} \pm \vec{v} \times \overrightarrow{\mathcal{B}}) \cdot \nabla_{p} f_{ \pm} \mp \frac{M^{\star}}{E_{p}} \nabla_{x} M^{\star} \cdot \nabla_{p} f_{ \pm} \\
& \quad=0
\end{aligned}
$$

where $\vec{v}=\vec{p} / E_{p}$ and

$$
\begin{aligned}
\mathcal{E}_{i}^{(j)} & =\mathcal{F}_{0 i}^{(j)} \mp \frac{M_{j}^{\star}(x)}{E_{p}^{(j)}} \nabla_{x, i} M_{j}^{\star}(x), \quad j=p, n, \\
\mathcal{E}_{i}^{(e)} & =\mathcal{F}_{0 i}^{(e)}, \\
\mathcal{B}_{i}^{(j)} & =\epsilon_{i l m} \partial_{x, l} \mathcal{V}_{m}^{(j)}, \quad j=p, n, e,
\end{aligned}
$$

$i, l, m=1,2,3$ and

$$
f_{ \pm}(x, \vec{p})=\int d p_{0} F_{0 \pm}(x, p)=\int d p_{0} \operatorname{Tr}\left[\gamma^{0} \hat{W}_{4}^{( \pm)(0)}\right] .
$$

An important remark is in order: The Vlasov equation can be derived in other approaches, for instance, in Refs. [20,21], this equation has been obtained using a Hamiltonian formalism. The main advantage of the Wigner approach is that one has a systematic method for obtaining the particle equilibrium distribution functions, which is of fundamental importance for the description of systems in a magnetized medium.

\section{B. Wigner equilibrium function in a magnetized medium}

The equilibrium Wigner distribution function is defined as:

$$
f_{ \pm}^{(0)(j)}(x, \vec{p})=\frac{1}{(2 \pi)^{4}} \int d p_{0} \operatorname{Tr}\left[\hat{\rho} \gamma^{0} \hat{W}_{4}^{( \pm)(0)}\right],
$$

where the density matrix operator is given by:

$$
\hat{\rho}=\frac{1}{Z} e^{-\beta\left(\hat{H}-\sum_{j} \mu_{j} \hat{N}_{j}\right)},
$$

where $Z$ is the partition function, $\beta=1 / T$, and the trace is to be evaluated simultaneously in the Dirac space and in any convenient set of basis states, for example, the JohnsonLippmann [22]. Of course, $f_{ \pm}^{(0)}(x, \vec{p})$ satisfies the Vlasov equation by its construction. Substituting the explicit form of the Wigner operator, Eq. (6), and the on-mass-shell constraint, Eq. (11), one obtains:

$$
\begin{aligned}
& f_{ \pm}^{(0)(j)}(x, \vec{p})=\int \frac{d p_{0}}{(2 \pi)^{4}} \operatorname{Tr}\left[\hat{\rho} \gamma^{0} \int d^{4} y e^{-i p \cdot y}\right.
\end{aligned}
$$

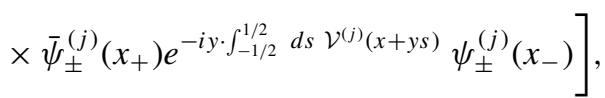

where $\psi_{ \pm}^{(j)}(x), j=(n, p, e)$, are the positive and negative components of the fermionic Dirac fields associated to the neutron, proton, and electron. From here on, we restrict our calculations to the mean-field approximation. Therefore, only the time component of the $\omega$ and $\rho$ vector meson fields are different from zero at equilibrium:

$$
V_{\mu}^{(0)}=V_{0} \delta_{\mu 0}, \quad b_{\mu}^{(0)}=b_{0} \delta_{\mu 0} .
$$

We choose the Landau gauge for the vector potential: $A_{\mu}^{(0)}=$ $B x_{2} \delta_{\mu 3}$, thus the external strong magnetic field is $\vec{B}=B \hat{e}_{3}$ and $\nabla \cdot \vec{B}=0$. The integral in the phase factor, Eq. (17), can be easily evaluated resulting in $\mathcal{V}_{\mu}^{(0)(j)}$, thus:

$$
\begin{aligned}
& f_{ \pm}^{(0)(j)}(x, \vec{p}) \\
& \quad=\int \frac{d p_{0}}{(2 \pi)^{4}} \int d^{4} y \operatorname{Tr}\left[\hat{\rho} \gamma^{0} e^{-i\left(p+\mathcal{V}^{(0)(j)}\right) \cdot y} \bar{\psi}_{ \pm}^{(j)}\left(x_{+}\right) \psi_{ \pm}^{(j)}\left(x_{-}\right)\right] .
\end{aligned}
$$

For the calculation of the equilibrium function, one has to insert the Dirac field operator in the Fock space in the last expression and to perform the integrals indicated in Eq. (19). A similar calculation has been done in Ref. [16] for a magnetized electron gas and can be promptly generalized for the present situation resulting in the following expression:

$$
\begin{aligned}
f_{ \pm}^{(0)(j)}(\vec{p})= & \sum_{n=0}^{\infty}\left[L_{n}\left(2 w^{2}\right)-L_{n-1}\left(2 w^{2}\right)\right] \\
& \times \frac{(-1)^{n} e^{-w^{2}}}{1+\exp \left[\beta\left(E_{n} \mp \tilde{\mu}^{(j)}\right)\right]}, \quad j=p, e,
\end{aligned}
$$

where $w^{2}=\frac{p_{1}^{2}}{e B}=\frac{p_{1}^{2}+p_{2}^{2}}{e B}, L_{n}(x)$ are the Laguerre polynomials, $\tilde{\mu}^{(e)}=\mu_{e}$, and $\tilde{\mu}^{(p)}=\mu_{p}-\mathcal{V}_{0}^{(0)(p)}$ are the effective chemical potential of the electron and proton, respectively, $E_{n}=\sqrt{p_{3}^{2}+M_{j}^{\star 2}+2 e B n}$ where $n$ is the Landau level label, and we have defined $L_{-1}(x)=0$. Since in this work we are only interested in systems at zero temperature, $T=0$, one can rewrite the last equation as:

$$
\begin{aligned}
f^{(0)(j)}(\vec{p})= & \sum_{n=0}^{\infty}\left[L_{n}\left(2 w^{2}\right)-L_{n-1}\left(2 w^{2}\right)\right] \\
& \times(-1)^{n} e^{-w^{2}} \theta\left(\tilde{\mu}^{(j)}-E_{n}\right),
\end{aligned}
$$

where the Heaviside function $\theta(x)$ was used. The limit $T \rightarrow 0$ rules out the negative energy part of the distribution function, 
hence we will omit from here on the redundant plus signal in the symbol $f_{+}^{(0)(j)}$. The electron or proton equilibrium densities can be readily calculated from their corresponding distribution functions. For example, the electron density reads from Eq. (21):

$$
\begin{aligned}
\rho_{e}^{(0)}= & \frac{2}{(2 \pi)^{3}} \int d^{3} p f^{(0)(e)}(\vec{p}) \\
= & \frac{2}{(2 \pi)^{3}} \int_{-\infty}^{\infty} d p_{\|} \int_{0}^{\infty} d p_{\perp} p_{\perp} \int_{0}^{2 \pi} d \Phi f^{(0)(e)}(\vec{x}, \vec{p}, t) \\
= & \frac{e B}{\pi^{2}} \sum_{n=0}^{\infty} \int_{0}^{\infty} d p_{\|} \int_{0}^{\infty} d w w\left[L_{n}\left(2 w^{2}\right)-L_{n-1}\left(2 w^{2}\right)\right] \\
& \times e^{-w^{2}}(-1)^{n} \theta\left(\mu_{e}-E_{n}\right) .
\end{aligned}
$$

The last expression can be easily calculated in cylindrical coordinates, since the integral in $w$ can be found in tables of integrals [23], and the integral in $p_{\|}=p_{3}$ is calculated using the Heaviside function. Therefore:

$$
\rho_{e}^{(0)}=\frac{e B}{2 \pi^{2}} \sum_{n=0}^{n_{\max }} g_{n} p_{F}^{(e)}(n),
$$

with $n_{\max }=\left[\frac{\mu_{e}^{2}-m_{e}^{2}}{2 e B}\right]$ where [...] stands for the floor function, which gives the greatest integer that is less than or equal to $x . g_{n}$ is a degeneracy factor, which is 1 for $n=0$ and 2 for $n \geqslant 0$, and $p_{F}^{(e)}(n)=\sqrt{\mu_{e}^{2}-m_{e}^{2}-2 e B n}$. The density of electrons just obtained coincides with the usual one [24]. The neutron distribution function is also the standard one: $f^{(0)(n)}(\vec{p})=\theta\left(p_{F}^{(n) 2}-\vec{p}^{2}\right)$. Notice that the normalization factor of the distribution functions are determined according to the definition of the currents as given in the next section.

\section{Dispersion relations}

The four-current is given by:

$$
J_{\mu}^{(j)}(x)=\frac{2}{(2 \pi)^{3}} \int \frac{d^{3} p}{p^{0}} p_{\mu}\left[f_{+}^{(j)}(x, \vec{p})-f_{-}^{(j)}(x, \vec{p})\right],
$$

where $j=(n, p, e)$ and $p^{0}=E_{j}=\sqrt{\vec{p}^{2}+M_{j}^{\star}}$. It follows from the Vlasov equation, Eq. (14), the following four-current conservation law: $\partial^{\mu} J_{\mu}^{(j)}(x)=0$.

The dispersion relations will be obtained considering a small perturbation from equilibrium of the distribution functions, where the equilibrium four-current is given by:

$$
J_{\mu}^{(0)(j)}=\frac{2}{(2 \pi)^{3}} \int \frac{d^{3} p}{p^{0}} p_{\mu}\left[f_{+}^{(0)(j)}(\vec{p})-f_{-}^{(0)(j)}(\vec{p})\right] .
$$

Next, we consider only the zero-temperature case, $T=0$. Hence, we can write:

$$
f^{(j)}(\vec{x}, \vec{p}, t)=f^{(0)(j)}(\vec{p})+\delta f^{(j)} .
$$

The small perturbation of the distribution functions, $f^{(0)(j)}$, around their equilibrium values, will generate perturbations on the fields:

$$
\begin{aligned}
\phi & =\phi^{(0)}+\delta \phi, \quad V_{\mu}=V_{\mu}^{(0)}+\delta V_{\mu}, \quad b_{\mu}=b_{\mu}^{(0)}+\delta b_{\mu}, \\
A_{\mu} & =A_{\mu}^{(0)}+\delta A_{\mu},
\end{aligned}
$$

and cause a corresponding perturbation of the equilibrium fourcurrent,

$$
J_{\mu}^{(j)}(x)=J_{\mu}^{(0)(j)}+\delta J_{\mu}^{(j)},
$$

with

$$
\delta J_{\mu}^{(j)}=\frac{2}{(2 \pi)^{3}} \int \frac{d^{3} p}{E_{j}^{(0)}} p_{\mu} \delta f^{(j)}
$$

where we have used the notation $E_{j}^{(0)}=\sqrt{\vec{p}^{2}+M_{j}^{\star(0)^{2}}}, j=$ $(n, p, e)$, with $M_{p}^{\star(0)}=M_{n}^{\star(0)}=M-g_{s} \phi^{(0)}$, and $M_{e}^{\star(0)}=m_{e}$. The scalar density is given by the expression:

$$
\rho_{s}^{(j)}=\frac{2}{(2 \pi)^{3}} \int \frac{d^{3} p}{E_{j}} M_{j}^{\star} f^{(j)}(x, \vec{p}) .
$$

The small perturbation of the proton and neutron scalar densities have to be calculated with care [25], since $M_{j}^{\star}=$ $M-g_{s} \phi(x)$ is position dependent, resulting in the following expression:

$$
\rho_{s}^{(j)}=\rho_{s}^{(0)(j)}+\delta \rho_{s},
$$

with

$$
\rho_{s}^{(0)(j)}=\frac{2}{(2 \pi)^{3}} \int \frac{d^{3} p}{E_{j}^{(0)}} M_{j}^{\star(0)} f^{(0)(j)}(\vec{p}),
$$

and $\delta \rho_{s}^{(j)}=\delta \tilde{\rho}_{s}^{(j)}+g_{s} d \rho_{s}^{(0)(j)} \delta \phi$, with:

$$
\delta \tilde{\rho}_{s}^{(j)}=\frac{2}{(2 \pi)^{3}} \int \frac{d^{3} p}{E_{j}^{(0)}} M_{j}^{\star(0)} \delta f^{(j)},
$$

and

$$
d \rho_{s}^{(0)(j)}=-\frac{2}{(2 \pi)^{3}} \int d^{3} p \frac{\vec{p}^{2}}{E_{j}^{(0)^{3}}} f^{(0)(j)} .
$$

After substituting Eq. (25) in the Vlasov equation, Eq. (14) retaining only terms of the first order in $\delta f^{(j)}$, one obtains:

$$
\begin{aligned}
\partial_{t} \delta f^{(j)}+\vec{v} \cdot \nabla_{x} \delta f^{(j)}+\vec{v} \times\left(\nabla_{x} \times \overrightarrow{\mathcal{V}}^{(0)(j)}\right) \cdot \nabla_{p} \delta f^{(j)} \\
+\left[\vec{v} \times \nabla_{x} \times\left(\overrightarrow{\mathcal{V}}^{(0)(j)}+\delta \overrightarrow{\mathcal{V}}^{(j)}\right)+g_{s} \frac{M_{j}^{\star(0)}}{E_{j}^{(0)}} \nabla_{x} \delta \phi\right. \\
\left.-\partial_{t} \delta \overrightarrow{\mathcal{V}}^{(j)}-\nabla_{x} \delta \mathcal{V}_{0}^{(j)}\right] \cdot \nabla_{p} f^{(0)(j)}=0
\end{aligned}
$$

where $\vec{v}=\vec{p} / E_{j}^{(0)}, j=p, e$ (for the electrons $g_{s}=0$ ). The last equation can be further simplified noting that the equilibrium distribution function, Eq. (21), is a function of $p_{\|}$and $p_{\perp}$ only, hence, it is useful to employ cylindrical coordinates $\left(p_{\perp}, \Phi, p_{\|}\right)$to rewrite Eq. (34) as:

$$
\begin{aligned}
\partial_{t} \delta f^{(j)}+\vec{v} \cdot \nabla_{x} \delta f^{(j)}-\frac{Q_{j} B}{E_{j}^{(0)}} \frac{\partial}{\partial \Phi} \delta f^{(j)} \\
+\left[\vec{v} \times\left(\nabla_{x} \times \delta \overrightarrow{\mathcal{V}}^{(j)}\right)+g_{s} \frac{M_{j}^{\star(0)}}{E_{j}^{(0)}} \nabla_{x} \delta \phi\right. \\
\left.-\partial_{t} \delta \overrightarrow{\mathcal{V}}^{(j)}-\nabla_{x} \delta \mathcal{V}_{0}^{(j)}\right] \cdot \nabla_{p} f^{(0)(j)}=0 .
\end{aligned}
$$


In the latter equation, $Q_{j}$ is the electric charge of the $j$ particle. Note also that this equation is also valid for the neutron, if one takes $Q_{n}=0$. Next, we obtain the dispersion relations, starting from the Fourier transform of the small deviation from equilibrium of the fields and of the distribution functions:

$$
\left\{\begin{array}{c}
\delta f^{(j)}(\vec{x}, \vec{p}, t) \\
\delta \phi(\vec{x}, t) \\
\delta \mathcal{V}_{\mu}^{(j)}(\vec{x}, t)
\end{array}\right\}=\int d^{3} q d \omega\left\{\begin{array}{c}
\delta f^{(j)}(\vec{q}, \omega, \vec{p}) \\
\delta \phi(\vec{q}, \omega) \\
\delta \mathcal{V}_{\mu}^{(j)}(\vec{q}, \omega)
\end{array}\right\} e^{i(\omega t-\vec{q} \cdot \vec{x})}
$$

and after substituting the last equation in the Vlasov equation, Eq. (35), one obtains for $\delta f^{(j)}(\vec{q}, \omega, \vec{p})$ :

$$
i(\omega-\vec{v} \cdot \vec{q}) \delta f^{(j)}-\frac{Q_{j} B}{E_{j}^{(0)}} \frac{\partial}{\partial \Phi} \delta f^{(j)}=i\left[(\omega-\vec{v} \cdot \vec{q}) \delta \overrightarrow{\mathcal{V}}^{(j)}-\left(\delta \mathcal{V}_{0}^{(j)}-\vec{v} \cdot \delta \overrightarrow{\mathcal{V}}^{(j)}-g_{s} \frac{M_{j}^{\star(0)}}{E_{j}^{(0)}} \delta \phi\right) \vec{q}\right] \cdot \nabla_{p} f^{(0)(j)}=0 .
$$

Here, we note that the last equation has an explicit dependence on the angle $\Phi$. In order to get rid of this angular dependence, we will use some techniques, which are well known in magnetized plasma physics $[26,27]$. We will adopt the same frame of reference used in Ref. [27] where:

$$
\begin{aligned}
& \vec{B}=B \vec{e}_{3}, \hat{e}_{3} \equiv e_{\|} \\
& \vec{q}=q_{\perp} \vec{e}_{1}+q_{\|} \vec{e}_{3}=\left(q_{\perp}, 0, q_{\|}\right) \\
& \vec{p}=p_{\perp} \vec{e}_{\perp}+p_{\|} \vec{e}_{3}=\left(p_{\perp} \cos \Phi, p_{\|} \sin \Phi, p_{\|}\right) .
\end{aligned}
$$

The angular dependence of $\delta f^{(j)}(\vec{q}, \omega, \vec{p})=\delta f^{(j)}\left(\vec{q}, \omega, p_{\perp}\right.$, $\left.\Phi, p_{\|}\right)$can be separated by using the Oberman-Ron [26] transform:

$$
\begin{aligned}
& \delta f^{(j)}(\vec{q}, \omega, \vec{p}) \\
& \quad=e^{i b \sin \Phi} \sum_{m=-\infty}^{\infty} e^{-i m \Phi} J_{m}(b) \delta f_{m}^{(j)}\left(\vec{q}, \omega, p_{\|}, p_{\perp}\right),
\end{aligned}
$$

and its inverse transform:

$$
\begin{aligned}
& \delta f_{m}^{(j)}\left(\vec{q}, \omega, p_{\|}, p_{\perp}\right) \\
& \quad=\frac{1}{2 \pi J_{m}(b)} \int_{0}^{2 \pi} d \Phi e^{-i b \sin \Phi} e^{i m \Phi} \delta f^{(j)}(\vec{q}, \omega, \vec{p}),
\end{aligned}
$$

where $J_{m}(b)$ is a Bessel function and $b$ is and arbitrary real constant. We substitute the Oberman-Ron transform, Eq. (39), in the Vlasov equation, Eq. (37), with $b=-\frac{q_{\perp} p_{\perp}}{Q_{j} B}, j=(p, e)$, and integrate in $\Phi$ the resulting expression multiplied by the factor exp $[-i(b \sin \Phi-m \Phi)]$. After some straightforward but tedious calculation, we finally obtain, using Eq. (40), the following expression:

$$
\begin{aligned}
\delta f^{(j)}(\vec{q}, \omega, \vec{p})= & e^{i b \sin \Phi} \sum_{m=-\infty}^{\infty} e^{-i m \Phi}\left\{\frac{m}{b} J_{m}(b)\left[\omega D_{\perp}^{(j)}-\frac{q_{\|}}{E_{j}^{(0)}}\left(p_{\|} D_{\perp}^{(j)}-p_{\perp} D_{\|}^{(j)}\right)\right] \delta \mathcal{V}_{x}^{(j)}\right. \\
& +i J_{m}^{\prime}(b)\left[\omega D_{\perp}^{(j)}-\frac{q_{\|}}{E_{j}^{(0)}}\left(p_{\|} D_{\perp}^{(j)}-p_{\perp} D_{\|}^{(j)}\right)\right] \delta \mathcal{V}_{y}^{(j)}+J_{m}(b)\left[\omega D_{\|}^{(j)}-\frac{q_{\perp}}{E_{j}^{(0)}}\left(p_{\perp} D_{\|}^{(j)}-p_{\|} D_{\perp}^{(j)}\right) \frac{m}{b}\right] \delta \mathcal{V}_{z}^{(j)} \\
& \left.-J_{m}(b)\left[q_{\perp} D_{\perp}^{(j)} \frac{m}{b}+q_{\|} D_{\|}^{(j)}\right]\left[\delta \mathcal{V}_{0}^{(j)}-\frac{g_{s} M_{j}^{\star(0)}}{E_{j}^{(0)}} \delta \phi\right]\right\}\left[\omega-\frac{p_{\|} q_{\|}}{E_{j}^{(0)}}+\frac{Q_{j} B m}{E_{j}^{(0)}}\right]^{-1}
\end{aligned}
$$

where the prime of the Bessel function means its derivative with respect to $b$, and

$$
D_{\perp}^{(j)}=\frac{\partial}{\partial p_{\perp}} f^{(0)(j)}, \quad D_{\|}^{(j)}=\frac{\partial}{\partial p_{\|}} f^{(0)(j)} .
$$

The above expression is the key for the calculation of the dispersion relations in the presence of a strong external magnetic field. The Fourier transform of the small deviations from equilibrium of the current is given by:

$$
\delta J_{\mu}^{(j)}(\vec{x}, t)=\int d^{3} q d \omega \delta J_{\mu}^{(j)}(\vec{q}, \omega) e^{i(\omega t-\vec{q} \cdot \vec{x})}
$$

From Eq. (28) it follows that:

$$
\delta J_{\mu}^{(j)}(\vec{q}, \omega)=\frac{2}{(2 \pi)^{3}} \int \frac{d^{3} p}{E_{j}^{(0)}} p_{\mu} \delta f^{(j)}(\vec{q}, \omega, \vec{p}),
$$

and the current conservation results in the relation:

$$
\partial^{\mu} J_{\mu}^{(j)}=0 \Rightarrow \omega \delta J_{0}^{(j)}+\vec{q} \cdot \delta \vec{J}^{(j)}=0 .
$$

The continuity equation also can be used to derive the important relation:

$$
\partial^{\mu} \delta \mathcal{V}_{\mu}^{(j)}=0 \Rightarrow \omega \delta \mathcal{V}_{0}^{(j)}+\vec{q} \cdot \delta \overrightarrow{\mathcal{V}}^{(j)}=0
$$




\section{Dispersion relations for longitudinal and transverse density modes}

The dispersion relation for density perturbations follows from taking $\mu=0$ in Eq. (44):

$$
\delta \rho^{(j)} \equiv \delta J_{0}^{(j)}(\vec{q}, \omega)=\frac{2}{(2 \pi)^{3}} \int d^{3} p \delta f^{(j)}(\vec{q}, \omega, \vec{p})=\frac{2}{(2 \pi)^{3}} \int_{0}^{\infty} d p_{\perp} p_{\perp} \int_{-\infty}^{\infty} d p_{\|} \int_{0}^{2 \pi} d \Phi \delta f^{(j)}(\vec{q}, \omega, \vec{p}) .
$$

Then, substituting Eq. (41) in the last expression and performing the integration in $\Phi$, one obtains:

$$
\begin{aligned}
& \delta \rho^{(j)}(\vec{q}, \omega) \\
& =S_{j}\left[\frac{m}{b} J_{m}^{2}(b)\left(\omega D_{\perp}^{(j)}-\frac{q_{\|}}{E_{j}^{(0)}}\left(p_{\|} D_{\perp}^{(j)}-p_{\perp} D_{\|}^{(j)}\right)\right)\right] \delta \mathcal{V}_{x}^{(j)}+S_{j}\left[i J_{m}(b) J_{m}^{\prime}(b)\left(\omega D_{\perp}^{(j)}-\frac{q_{\|}}{E_{j}^{(0)}}\left(p_{\|} D_{\perp}^{(j)}-p_{\perp} D_{\|}^{(j)}\right)\right)\right] \delta \mathcal{V}_{y}^{(j)} \\
& \quad+S_{j}\left[J_{m}^{2}(b)\left(\omega D_{\|}^{(j)}-\frac{q_{\perp}}{E_{j}^{(0)}}\left(p_{\perp} D_{\|}^{(j)}-p_{\|} D_{\perp}^{(j)}\right) \frac{m}{b}\right)\right] \delta \mathcal{V}_{z}^{(j)}-S_{j}\left[J_{m}^{2}(b)\left(q_{\perp} D_{\perp}^{(j)} \frac{m}{b}+q_{\|} D_{\|}^{(j)}\right)\right] \delta \mathcal{V}_{0}^{(j)} \\
& \quad+S_{j}\left[\frac{J_{m}^{2}(b)}{E_{j}^{(0)}}\left(q_{\perp} D_{\perp}^{(j)} \frac{m}{b}+q_{\|} D_{\|}^{(j)}\right)\right] g_{s} M_{j}^{\star(0)} \delta \phi
\end{aligned}
$$

where we have defined, as in Ref. [27], the function:

$$
S_{j}[X]=\sum_{m=-\infty}^{\infty} \frac{1}{2 \pi^{2}} \int_{-\infty}^{\infty} d p_{\|} \int_{0}^{\infty} \frac{d p_{\perp} p_{\perp}[X]}{\omega-\frac{p_{\|} q_{\|}}{E_{j}^{(0)}}+\frac{Q_{j} B m}{E_{j}^{(0)}}},
$$

where $X$ is an arbitrary function of $p_{\|}$and $p_{\perp}$. The next step in order to obtain the dispersion relations is to substitute the mesonic deviations from equilibrium in the last equation by using the equations of motion. An additional equation involving the scalar density is necessary to determine the dispersion relation. From Eq. (32), it follows analogously to the particle density fluctuations that:

$$
\delta \tilde{\rho}_{s}^{(j)}=\frac{2}{(2 \pi)^{3}} \int d^{3} p \frac{M_{j}^{\star^{(0)}}}{E_{j}^{(0)}} \delta f^{(j)}(\vec{q}, \omega, \vec{p}) .
$$

Following the same reasoning used in the derivation of Eq. (48), one obtains an analogous expression for the scalar density in terms of the meson fields and the generalized Lindhard function. In fact, the expression for $\delta \tilde{\rho}_{s}^{(j)}$ can be read from Eq. (48) by including $M_{j}^{\star(0)} / E_{j}^{(0)}$ in the integrands. In the Appendix, we show, using the equations of motion for the meson and electromagnetic fields, that one can write the deviations from the equilibrium of these fields in terms of the deviations of the corresponding densities. Therefore, substituting these field deviations in Eq. (48), one obtains the dispersion relations.

\section{Longitudinal mode}

The longitudinal mode corresponds to small perturbations parallel to the magnetic field and the dispersion relations are obtained by taking $q_{\perp}=0, q_{\|}=q, b=0$ and $\delta \mathcal{V}_{x}=\delta \mathcal{V}_{y}=0$ in Eq. (48). From the conservation law, Eq. (46), $\partial^{\mu} \mathcal{V}_{\mu}^{(j)}=0$, for the longitudinal modes we find:

$$
\omega \delta \mathcal{V}_{0}^{(j)}=\vec{q} \cdot \delta \overrightarrow{\mathcal{V}}^{(j)}=q \delta \mathcal{V}_{z}^{(j)}
$$

The last result allows us to write $\delta \mathcal{V}_{z}^{(j)}$ in terms of $\delta \mathcal{V}_{0}^{(j)}$, hence $\delta \rho^{(j)}(q, \omega)$ in Eq. (48) reduces to:

$$
\begin{aligned}
\delta \rho^{(j)}(\vec{q}, \omega)= & -q\left(1-\frac{\omega^{2}}{q^{2}}\right) S_{j}\left[J_{m}^{2}(0) D_{\|}^{(j)}\right] \delta \mathcal{V}_{0}^{(j)} \\
& +q S_{j}\left[\frac{J_{m}^{2}(0)}{E_{j}^{(0)}} D_{\|}^{(j)}\right] g_{s} M_{j}^{\star(0)} \delta \phi .
\end{aligned}
$$

In a completely analogous way, one obtains for the scalar density:

$$
\begin{aligned}
\delta \tilde{\rho}_{s}^{(j)}(q, \omega)= & -q\left(1-\frac{\omega^{2}}{q^{2}}\right) S_{j}\left[\frac{J_{m}^{2}(0)}{E_{j}^{(0)}} D_{\|}^{(j)}\right] M_{j}^{\star(0)} \delta \mathcal{V}_{0}^{(j)} \\
& +q S_{j}\left[\frac{J_{m}^{2}(0)}{E_{j}^{(0)^{2}}} D_{\|}^{(j)}\right] g_{s} M_{j}^{\star(0)^{2}} \delta \phi
\end{aligned}
$$

From Eqs. (49), (52), (53), and the equations relating the perturbations of the mesonic fields with the corresponding perturbations of the currents given in the Appendix, Eqs. (A1), one obtains the dispersion relations. We have a set of five equations involving the number densities, $\delta \rho^{(p)}, \delta \rho^{(n)}, \delta \rho^{(e)}$, and the scalar densities $\delta \tilde{\rho}_{s}^{(p)}, \delta \tilde{\rho}_{s}^{(n)}$, resulting in the following matrix:

$$
\left(\begin{array}{ccccc}
a_{11} & a_{12} & a_{13} & a_{14} & a_{15} \\
a_{21} & a_{22} & 0 & a_{24} & a_{25} \\
a_{31} & 0 & a_{33} & 0 & 0 \\
a_{41} & a_{42} & a_{43} & a_{44} & a_{45} \\
a_{51} & a_{52} & 0 & a_{54} & a_{55}
\end{array}\right)\left(\begin{array}{l}
\delta \rho^{(p)} \\
\delta \rho^{(n)} \\
\delta \rho^{(e)} \\
\delta \tilde{\rho}_{s}^{(p)} \\
\delta \tilde{\rho}_{s}^{(n)}
\end{array}\right)=0 .
$$

The eigenmodes $\omega$ of the system are the solutions that correspond to the roots of the determinant of the latter matrix. In the Appendix, a more detailed discussion and the explicit expressions for the elements of the dispersion matrix are given. 

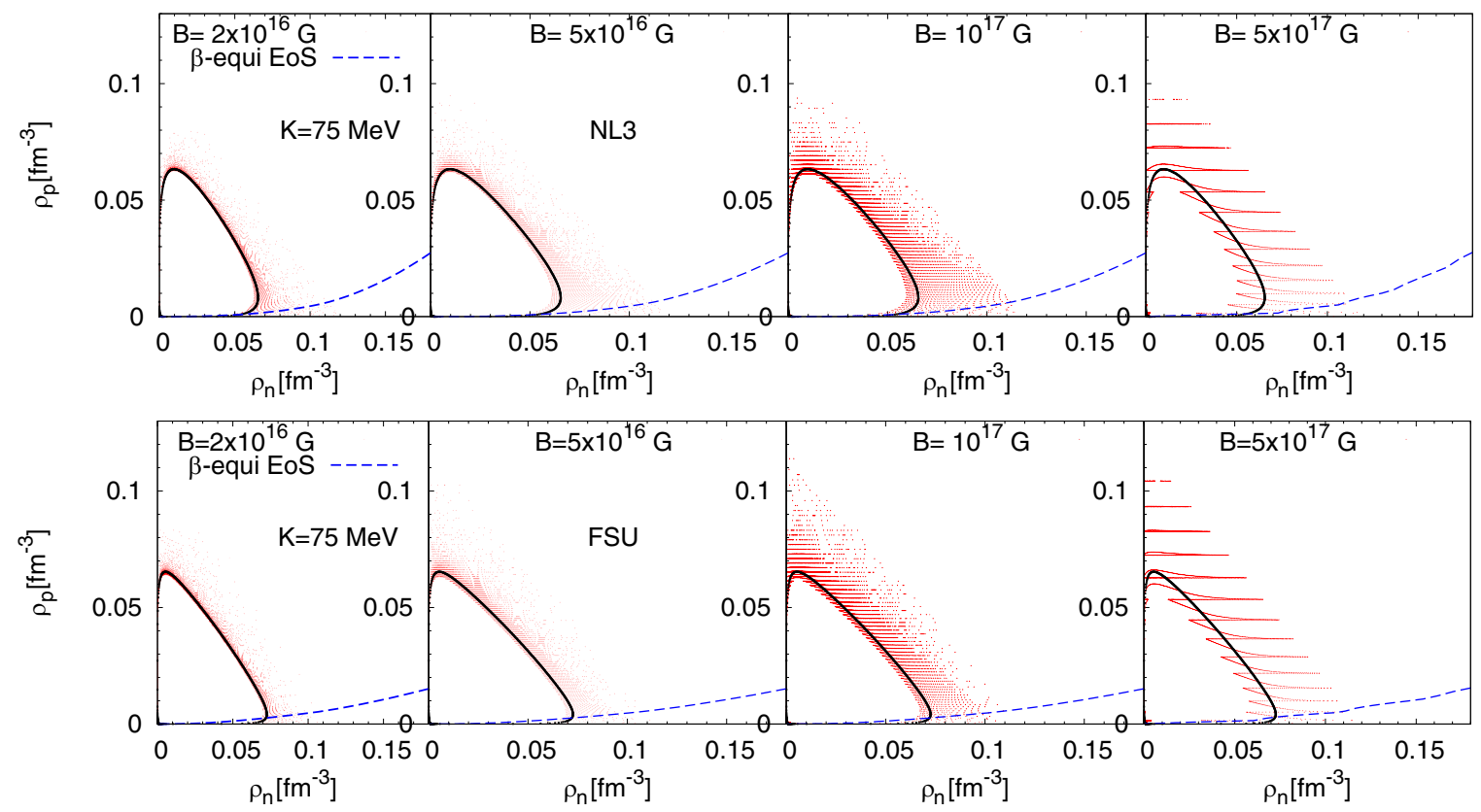

FIG. 1. Dynamical spinodals for the NL3 (top) and the FSU (bottom) parametrization, for several values of the magnetic field and for a moment transfer $k=75 \mathrm{MeV}$. The black curve corresponds to the $B=0$ results.

\section{Transverse mode}

The transverse mode corresponds to perturbations on the plane perpendicular to the direction of the external magnetic field. The dispersion relations are obtained by taking $q_{\perp}=\mathrm{q}$, $q_{\|}=0$, and $\delta \mathcal{V}_{y}=\delta \mathcal{V}_{z}=0$ in Eq. (48).

Proceeding as in the longitudinal mode, one obtains:

$$
\begin{aligned}
\delta \rho^{(j)}(q, \omega)= & -q\left(1-\frac{\omega^{2}}{q^{2}}\right) S_{j}\left[J_{m}^{2}(b) D_{\perp}^{(j)} \frac{m}{b}\right] \delta \mathcal{V}_{0}^{(j)} \\
& +q S_{j}\left[\frac{J_{m}^{2}(b)}{E_{j}^{(0)}} \frac{m}{b} D_{\perp}^{(j)}\right] g_{s} M^{\star(0)} \delta \phi
\end{aligned}
$$

and

$$
\begin{aligned}
\delta \tilde{\rho}_{s}^{(j)}(q, \omega)= & -q\left(1-\frac{\omega^{2}}{q^{2}}\right) S_{j}\left[\frac{J_{m}^{2}(b)}{E_{j}^{(0)}} D_{\perp}^{(j)} \frac{m}{b}\right] M_{j}^{\star(0)} \delta \mathcal{V}_{0}^{(j)} \\
& +q S_{j}\left[\frac{J_{m}^{2}(b)}{E_{j}^{(0)^{2}}} \frac{m}{b} D_{\perp}^{(j)}\right] g_{s} M_{j}^{\star(0)^{2}} \delta \phi .
\end{aligned}
$$

The dispersion relations for the transverse mode have the same matrix structure of Eq. (54), and the appropriate matrix elements for this case are discussed in detail in the Appendix.

\section{RESULTS}

In the present section, we discuss the effects of strong magnetic fields on the structure of the inner crust of magnetars by analyzing the dynamical spinodals for NL3 and FSU parametrizations. We consider the propagation of both longitudinal and transverse modes, and define the spinodal as the locus of the zero-frequency solutions of the dispersion relation obtained for each type. The two parametrizations NL3 and FSU will be considered because they represent two very distinct types of models: they have, respectively, a hard and a soft symmetry energy. We will also calculate the maximum growth rates in the interior of the spinodal surface and the corresponding wavelengths. All calculations are carried out at zero temperature, and without taking in account the anomalous magnetic moments (AMM) of nucleons. We will mostly consider magnetic field intensities between $B=2 \times 10^{16} \mathrm{G}$ and $B=5 \times 10^{17} \mathrm{G}$, although we will also show some results for $B \approx 5 \times 10^{18} \mathrm{G}$. The most intense fields detected on the surface of a magnetar are not larger than $2 \times 10^{15} \mathrm{G}$, however, toroidal fields more intense than $10^{17} \mathrm{G}$ in stable configurations were obtained in Refs. [28,29], and, therefore, one may expect stronger fields in the interior of the stars.

\section{A. Longitudinal modes and associated spinodals}

In Fig. 1, we show the dynamical spinodal sections in the $\left(\rho_{n}, \rho_{p}\right)$ space for magnetic field intensities $B=2 \times$ $10^{16} \mathrm{G}, B=5 \times 10^{16} \mathrm{G}, B=10^{17} \mathrm{G}$, and $B=5 \times 10^{17} \mathrm{G}$, obtained with the NL3 (top panels) and FSU (bottom panels) parametrizations. The black lines represent the spinodal section at zero magnetic field. The calculations were performed with $k=75 \mathrm{MeV}$, which is a value of the transferred momentum that gives a spinodal section very close to the envelope of the spinodal sections. Essentially, the size of the spinodal section does not change much for $70<k<100 \mathrm{MeV}$. In the figure, we also include the $\operatorname{EoS}$ of $\beta$-equilibrium matter represented by the blue dashed line. This will allow us to estimate the crust-core transition density from the crossing of the $\beta$-equilibrium EoS of stellar matter with the dynamical spinodal.

The spinodal sections have been obtained numerically by solving the dispersion relation for $\omega=0$. This was performed 

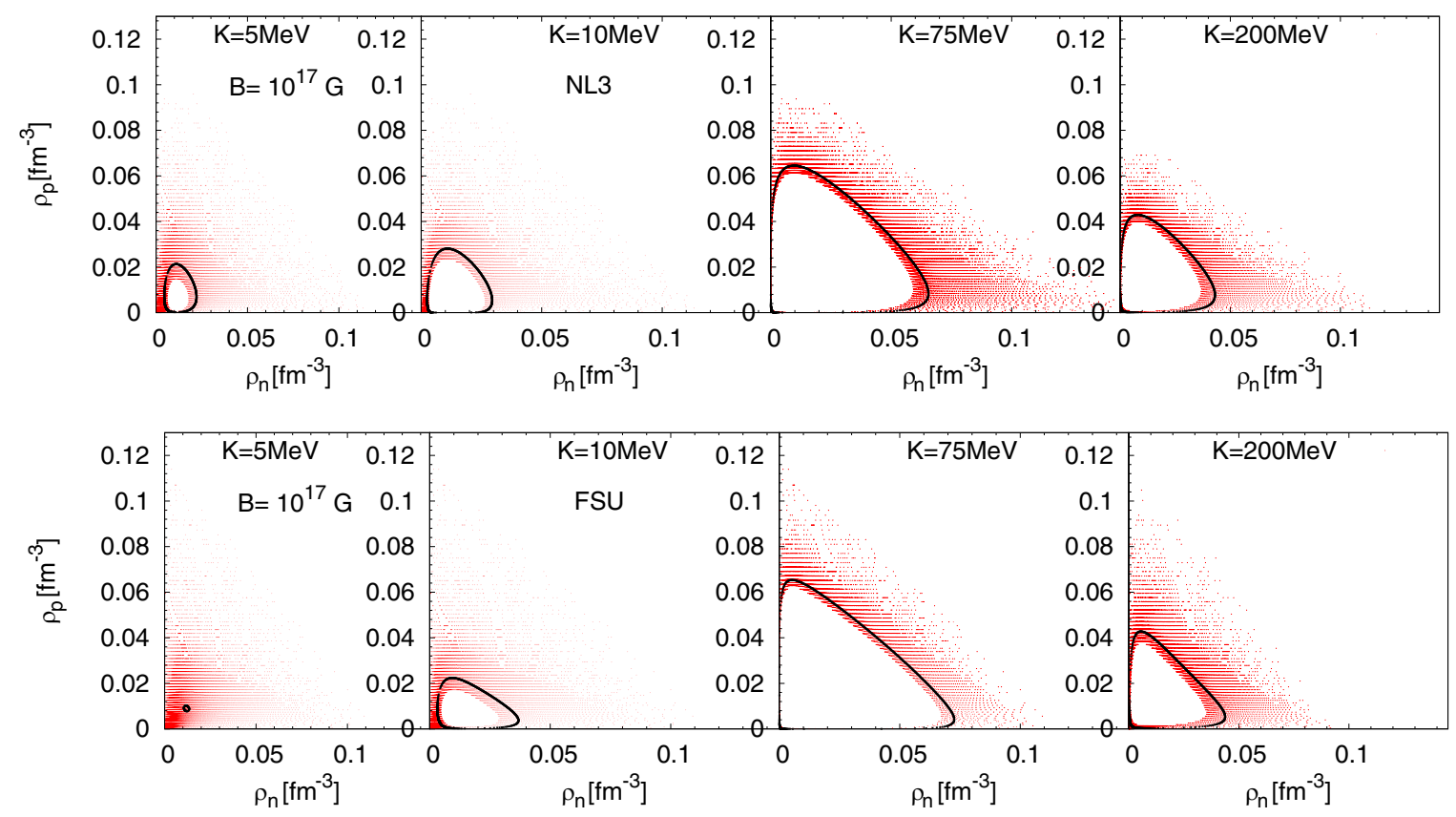

FIG. 2. Dynamical spinodals for the NL3 (top) and the FSU (bottom) parametrizations, for different moment transfer $k$ and for magnetic field $B=10^{17} \mathrm{G}$. The black curve corresponds to the $B=0$ results.

by looking for the solutions at a fixed proton fraction and, for each solution, a point was obtained. The solutions form a large connected region for the lower proton and neutron densities, plus extra disconnected domains that do not occur at $B=0$. The spinodal sections appear made of points, which, however, define close regions. The pointlike appearance of the sections is due to a numerical limitation. A higher resolution in $\left(\rho_{n}, \rho_{p}\right)$ would complete the gaps. Similar results have been obtained in Ref. [5].

The structure of the spinodal section obtained for the strongest field considered, $B=5 \times 10^{17}$, clearly shows the effect of the Landau quantization, as already shown in Ref. [5]: there are instability regions that extend to much larger densities than the $B=0$ spinodal section, while there are also stable regions that at $B=0$ would be unstable. This is due to the fact that the energy density becomes softer just after the opening of a new Landau level, and harder when the Landau level is most filled. For weaker fields, the same structure is found, but at a much smaller scale, due to the increase of the number of Landau levels; see for $B=10^{17}, B=5 \times 10^{16}$, and $B=2 \times 10^{16}$. The spinodal section tends to the $B=0$ one, as the magnetic field intensity is reduced. The results obtained with the present approach coincide with the ones obtained without the AMM in Ref. [5]. The present approach, however, will allow us to define the propagation of modes in an arbitrary direction with respect to the magnetic field direction. In the next section, we will discuss transverse modes.

Figure 1 allows a comparison of the spinodals obtained for two models with a very different behavior of the symmetry energy: the symmetry energy slope at saturation is $118 \mathrm{MeV}$ for NL3, and $60 \mathrm{MeV}$ for FSU. As discussed in previous works $[5,6,30]$, in the low proton density, the NL3 spinodal extends to larger neutron densities than FSU. On the other hand, at low neutron densities, the contrary occurs for FSU. These behaviors reflect the symmetry energy properties of both models: the larger NL3 slope at saturation density implies a smaller symmetry energy than FSU at densities below $\rho \approx$ $0.1 \mathrm{fm}^{-3}$, and this energetically favors asymmetric matter in NL3 with respect to FSU in that range of densities. Above $\rho \approx 0.1 \mathrm{fm}^{-3}$, it is FSU that favors more asymmetric matter, and this explains the behavior of the spinodals for low neutron densities, when many Landau levels are occupied by protons. For low proton densities, only the first Landau level is being occupied, and the spinodal extends to larger neutron densities for NL3 with a large symmetry energy slope [5,30,31].

In Fig. 2, we analyze the effect of the wave number on the extension of the spinodal. As discussed in Refs. [8,32], the extension of the spinodal increases when the wave number increases from zero to a value of the order of $\approx 70-100 \mathrm{MeV}$, due to the effect of the Coulomb field. We see that, for all the values of the transferred momentum, $k$, the disconnected regions are present, and have a similar extension, i.e., the upper limits of the disconnected region seem to be $k$ independent. This can be understood if one associates the isolated regions of instability to the onset of a new Landau level, which gives a particular extra stability as discussed in Ref. [31].

For very long wavelengths, the electrons and protons have to stick together in order to minimize the free energy, and this reduces a lot the unstable region. If $B=0$, and for a model with a large slope $L$, an unstable region at $k \approx 0$ still survives in the presence of electrons, however, this is not anymore true for a model with a soft symmetry energy such as FSU, for which there is no unstable region. At finite $B$, the effect of the Landau levels is important, and regions of instability arise in a large region of the phase space, even for small wave numbers. However, taking into account the discussion 

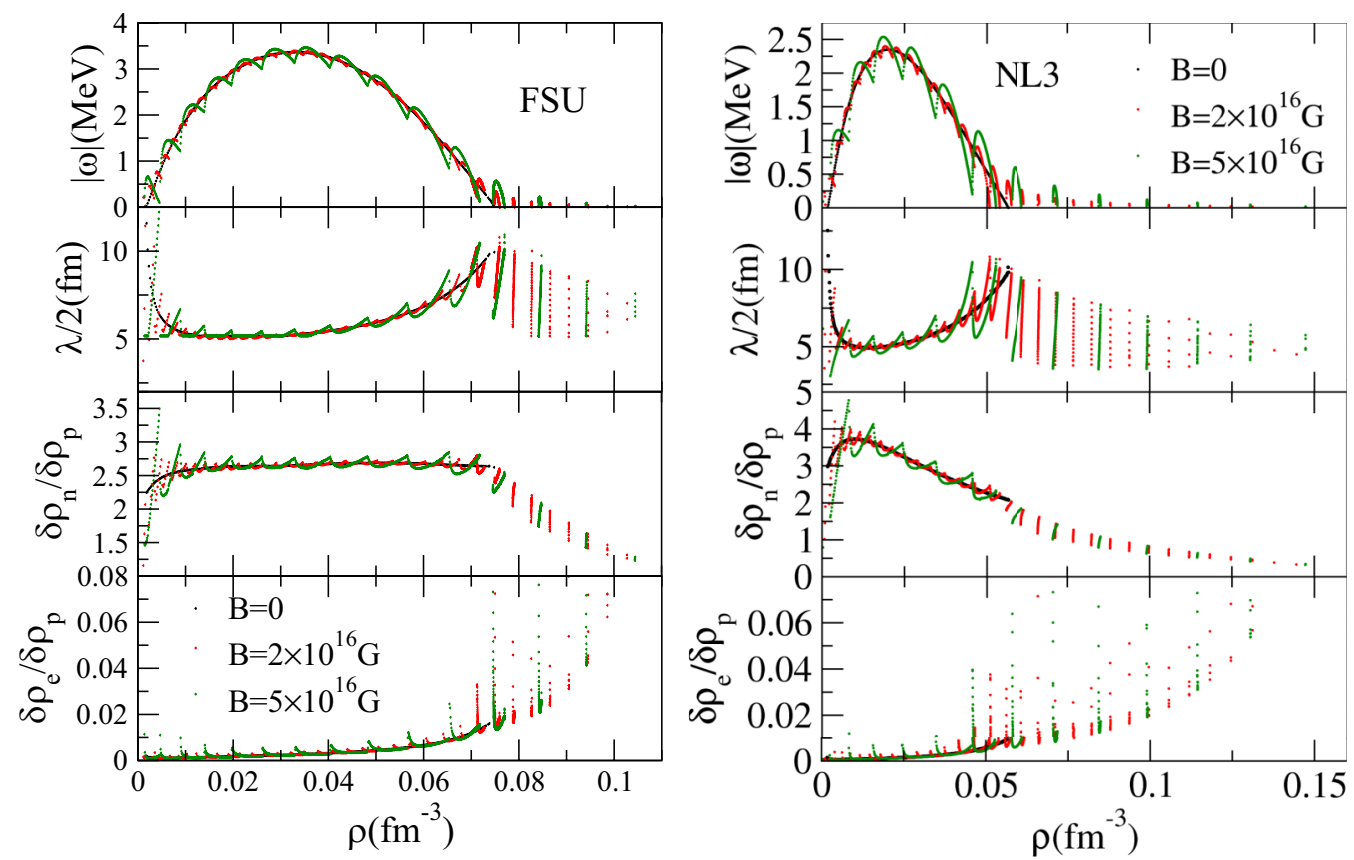

FIG. 3. Largest growth rate $\Gamma=|\omega|$ (top), the corresponding half-wavelength (middle), the neutron-proton density fluctuation ratio versus density, and the electron-proton density fluctuation ratio (bottom) versus density for different magnetic-field intensities and matter with $y_{p}=$ 0.035 for FSU (left), and with $y_{p}=0.02$ for NL3 (right). The black curve corresponds to the $B=0$ results.

of Ref. [7], it is expected that most of these effects are washed out for temperatures of the order of $\approx 0.5-1 \mathrm{MeV}$. At large wave numbers, $k>100 \mathrm{MeV}$, the spinodal region reduces due to the finite range of the nuclear force, as discussed in Ref. [8].

We will complete our discussion on the instabilities that arise due to the excitation of longitudinal modes, by calculating the largest growth rates for each density in the unstable region. The corresponding modes are the ones that most probably drive the system into a nonhomogeneous configuration. We consider that half the wavelength associated to these modes defines the average size of the clusters that are formed [33,34], as discussed in Refs. [5,6].

In Fig. 3, the largest growth rates (top panels), the corresponding half wavelength (second panels), the ratio $\delta \rho_{n} / \delta \rho_{p}$ between the neutron and proton density fluctuations (third panels), and the ratio $\delta \rho_{e} / \delta \rho_{p}$ between the electron and proton density fluctuations (bottom panels) are shown for two values of magnetic fields $B=2 \times 10^{16} \mathrm{G}$ (red) and $5 \times 10^{16} \mathrm{G}$ (green), and for the NL3 and FSU models. For each one, the calculation is done respectively at $y_{p}=0.02$ (NL3), and $y_{p}=0.035$ (FSU), which are the average proton fractions obtained in a Thomas-Fermi calculation of the pasta phases close to the crust-core transition [35]. In all figures, the $B=0$ results are plotted by a black curve. The results presented are equivalent to the ones given in Fig. 4 of Ref. [6] for the NL3 $\omega \rho$ model, excluding the AMM contribution. As discussed there, the plots present two well-defined density regions: a low-density region where the different quantities fluctuate around the $B=0$ results, the deviations being larger for the larger fields, and a region at higher density, with no instabilities
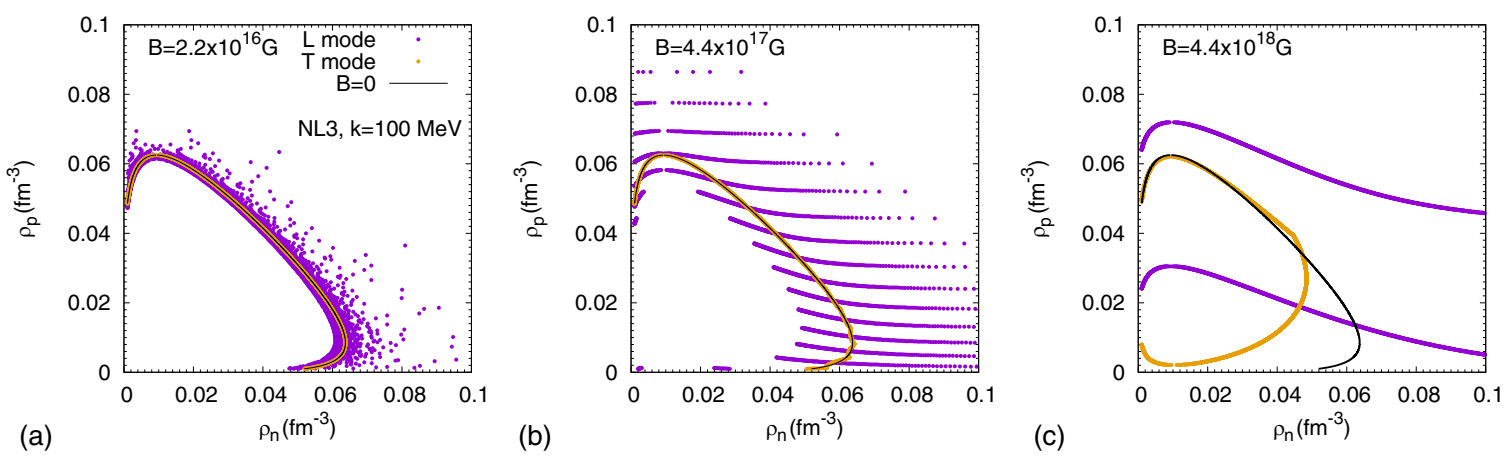

FIG. 4. Dynamical spinodals for npe matter subjected to an external magnetic field. The spinodal sections for both longitudinal (blue) and transverse modes (green) are given for $k=100 \mathrm{MeV}$ and (a) $B=2.2 \times 10^{16} \mathrm{G}$, (b) $B=4.4 \times 10^{17} \mathrm{G}$, (c) $B=4.4 \times 10^{18} \mathrm{G}$. The black curve corresponds to the $B=0$ results. 
at $B=0$, but that, at $B>0$, present regions of instability intercalated with stable regions. As expected from the spinodal regions plotted in Fig. 1, the second region extends to larger neutron densities for FSU, while the first region is smaller for the NL3 model. Otherwise, as discussed in Ref. [6], in the second region, the clusters become smaller, and more proton rich, as the density increases. In the bottom panel of Fig. 3, the ratio $\delta \rho_{e} / \delta \rho_{p}$ indicates how independent are the proton and electron fluctuations. For all the densities shown, it is clear that electron fluctuations are much smaller than the proton ones, and the system is far from a scenario where electrons have to fluctuate in phase with protons to lower the Coulomb energy, and which occurs for small wave numbers.

\section{B. Transverse modes}

In the previous section, only longitudinal modes have been considered. In the presence of strong magnetic fields, the motion of charged particles is favored along the magnetic field direction, and, therefore, it is expected that this is reflected on the spinodal region obtained from transverse modes. In Fig. 4, we plot the solutions of the dispersion relations for $\omega=$ 0 , for both longitudinal and transverse modes. We consider three different strengths of the external magnetic field: $B=$ $2.2 \times 10^{16} \mathrm{G}, B=4.4 \times 10^{17} \mathrm{G}$, and $B=4.4 \times 10^{18} \mathrm{G}$. The transferred momentum $k$ has been fixed at $100 \mathrm{MeV}$. The present comparison is undertaken only for the NL3 model (only values with $\rho_{n} \leqslant 0.1 \mathrm{fm}^{-3}$ are shown). For FSU we obtain similar results. Some conclusions are in order: for the most intense field considered, the spinodal connected to the longitudinal modes has two well-defined regions connected to the filling of the two Landau levels. As seen before, the spinodal extends to quite large neutron densities. A completely different effect is observed for the spinodal connected to the transverse modes. In this case, the very strong field $B=4.4 \times 10^{18}$ $G$ gives rise to a reduction of the spinodal at large neutron densities and isospin asymmetries. The magnetic field hinders the propagation of perturbations in a direction perpendicular to the magnetic field. However, for a magnetic field one order of magnitude smaller, this effect is washed out, and the transverse mode spinodal almost coincides with the $B=0$ spinodal. For this field intensity, the longitudinal mode spinodal is still far from the $B=0$ limit.

\section{CONCLUSIONS}

In the present work, we have applied the covariant formulation of the Vlasov equation presented in Ref. [12] to hadronic matter described within a relativistic mean-field approach. Within this formalism, we have calculated the normal modes of a relativistic plasma, having in mind the study of magnetized nuclear matter as found inside magnetars. The dispersion relations for both longitudinal and transverse density fluctuations were determined for nuclear matter described within a relativistic mean-field nuclear model with constant coupling constants.

In order to study the influence of the magnetic field on the extension of the crust of a magnetized star, we have calculated the unstable modes for two different nuclear models, which were chosen for their different density dependence of the symmetry energy. The spinodal section, the locus of the zero-frequency modes, was determined for the longitudinal and transverse density modes. We have also studied the growth rates of the unstable modes inside the spinodal sections, obtained by the largest growth rates, e.g., the modes that most probably determine the evolution of the system into a nonhomogeneous phase, and discussed the effect of the magnetic field on the size of the crust and on the size and proton content of the clusters formed in the inner crust of the star.

We have recovered previous results obtained for the longitudinal mode spinodals and corresponding unstable modes obtained with a generating function method. In particular, the effect of the magnetic field on the crust-core transition inside a neutron star was discussed. As already discussed before, it was shown that this transition occurs at larger densities, more strikingly if the slope of the symmetry energy is large, and that the transition region has a finite density width and is characterized by alternating stable and unstable regions.

However, within the formalism developed in the present study, we were able to study the propagation of modes not necessarily aligned with the magnetic field, and this was not possible in the approach of Ref. [5]. As an example, we have discussed the spinodal region that originates from the excitation of transverse modes. For magnetic fields of the order of $B \approx 5 \times 10^{18} \mathrm{G}$, it was shown that the spinodal section is much smaller than the $B=0$ section in the low proton density range. We found, however, that this effect is already washed out for a field one order of magnitude smaller, and that for $B<5 \times 10^{17} \mathrm{G}$, the transverse mode spinodal essentially coincides with the $B=0$ section.

Within the formalism developed, we aim at studying transport coefficients, e.g., heat and electrical conductivities and viscosity coefficients, of magnetized nuclear matter inside neutron stars. This are important quantities to determine the magnetic field evolution [36], the cooling of the star, or to understand the gravitational driven $r$-mode instabilities [37,38]. Another problem of interest for neutron stars is the study of the effect of the magnetic field on the nonhomogeneous matter that constitutes the inner crust, in particular, its effect on the shape of the clusters and orientation of the pasta phases. Although the dynamical spinodal includes the effect of surface tension (finite range of the force) and the Coulomb field, it does not take into account adequately the geometry, which is essential in this frustration problem of interplay between the Coulomb force and the surface tension. However, in the extended crust, we are dealing with quite dense matter, and it was seen that the wavelengths associated with the most unstable mode decrease outside the $B=0$ spinodal region. This may indicate that there will form thin tubes or deformed bubbles. In other systems that present pastalike phases, such as the surfactant liquid crystals, it has been shown that a magnetic field may induce orientational phase transitions [39].

\section{ACKNOWLEDGMENTS}

This work was supported by Fundação para a Ciência e Tecnologia, Portugal, under the projects UID/FIS/04564/2016 and POCI-01-0145-FEDER-029912 with financial support 
from POCI, in its FEDER component, and by the FCT/MCTES budget through national funds (OE). Partial support comes from PHAROS COST Action CA16214, and from Conselho Nacional de Desenvolvimento Científico e Tecnológico (CNPq) Grant No. 6484/2016-1, and as a part of the project
INCT-FNA [Instituto Nacional de Ciência e Tecnologia - Física Nuclear e Aplicaçõe (INCT-FNA)] 464898/2014-5 (S.A.), and Coordenação de Aperfeiçoamento de Pessoal de Nivel Superior (CAPES) (B.P.B.). H.P. is supported by FCT (Portugal) under Project No. SFRH/BPD/95566/2013.

\section{APPENDIX A: EQUATIONS OF MOTION FOR THE MESON AND ELECTROMAGNETIC FIELDS}

The equations of motion for the mesons and electromagnetic fields follow from using the Euler-Lagrangian equations (3):

$$
\begin{aligned}
\partial_{t}^{2} \phi-\nabla^{2} \phi+m_{s}^{2} \phi+\frac{\kappa}{2} \phi^{2}+\frac{\lambda}{6} \phi^{3} & =g_{s} \sum_{j=p, n} \rho_{s}^{(j)} \\
\partial_{t}^{2} V_{\mu}-\nabla^{2} V_{\mu}+m_{v}^{2} V_{\mu}+\frac{\xi}{6} V_{\nu} V^{\nu} V_{\mu}+2 \Lambda_{v} b_{\nu} b^{v} V_{\mu} & =g_{v} \sum_{j=p, n} J_{\mu}^{(j)} \\
\partial_{t}^{2} b_{\mu}-\nabla^{2} b_{\mu}+m_{\rho}^{2} b_{\mu}+2 \Lambda_{v} V_{v} V^{v} b_{\mu} & =\frac{g_{\rho}}{2} \sum_{j=p, n} \tau_{j} J_{\mu}^{(j)} \\
\partial_{t}^{2} A_{\mu}-\nabla^{2} A_{\mu}=e\left(J_{\mu}^{(p)}-J_{\mu}^{(e)}\right) & =\sum_{j=p, e} Q_{j} J_{\mu}^{(j)} .
\end{aligned}
$$

Now, we consider small deviations from the equilibrium in the fields, as given in Eq. (26), and perform a Fourier transform, obtaining:

$$
\begin{aligned}
& {\left[-\omega^{2}+\vec{q}^{2}+\tilde{m}_{s}^{2}\right] \delta \phi(\vec{q}, \omega)=g_{s} \sum_{j=p, n} \frac{2 M_{j}^{\star(0)}}{(2 \pi)^{3}} \int \frac{d^{3} p}{E_{j}^{(0)}} \delta f^{(j)}} \\
& {\left[-\omega^{2}+\vec{q}^{2}+m_{v}^{2}+\frac{\xi}{6} V_{0}^{(0)^{2}}+2 \Lambda_{v} b_{0}^{(0)^{2}}\right] \delta V_{\mu}+\frac{\xi}{3} V_{0}^{(0)^{2}} \delta V_{0} \delta_{\mu 0}+4 \Lambda_{v} V_{0}^{(0)} b_{0}^{(0)} \delta b_{0} \delta_{\mu 0}=g_{v} \sum_{j=p, n} \frac{2}{(2 \pi)^{3}} \int \frac{d^{3} p}{E_{j}^{(0)}} p^{\mu} \delta f^{(j)},} \\
& {\left[-\omega^{2}+\vec{q}^{2}+m_{\rho}^{2}+2 \Lambda_{v} V_{0}^{(0)^{2}}\right] \delta b_{\mu}+4 \Lambda_{v} V_{0}^{(0)} b_{0}^{(0)} \delta V_{0} \delta_{\mu 0}=\frac{g_{\rho}}{2} \sum_{j=p, n} \tau_{j} \frac{2}{(2 \pi)^{3}} \int \frac{d^{3} p}{E_{j}^{(0)}} p^{\mu} \delta f^{(j)},} \\
& {\left[-\omega^{2}+\vec{q}^{2}\right] \delta A_{\mu}=\sum_{j=p, e} Q_{j} \frac{2}{(2 \pi)^{3}} \int \frac{d^{3} p}{E_{j}^{(0)}} p^{\mu} \delta f^{(j)}}
\end{aligned}
$$

where $\tau_{j}= \pm 1$ is the isospin projection for protons and neutrons, respectively, and the effective scalar mass is given by:

$$
\tilde{m}_{s}^{2}=m_{s}^{2}+\kappa \phi^{(0)}+\frac{\lambda}{2} \phi^{(0)^{2}}-g_{s}^{2} \sum_{j=p, n} d \rho_{s}^{(0)(j)} .
$$

\section{APPENDIX B: DISPERSION RELATION MATRIX ELEMENTS FOR LONGITUDINAL MODES}

For the calculation of the dispersion relations for the longitudinal modes, Eqs. (52), (53), we first obtain $S_{j}$, by taking the derivative of the equilibrium distribution function, Eq. (21),

$$
D_{\|}^{(j)}=\frac{\partial}{\partial p_{\|}} f^{(0)(j)}=\sum_{n=0}^{\infty}\left[L_{n}\left(2 w^{2}\right)-L_{n-1}\left(2 w^{2}\right)\right](-1)^{n} e^{-w^{2}}\left\{-\delta\left[p_{\|}-p_{F}^{(j)}(n)\right]+\delta\left[p_{\|}+p_{F}^{(j)}(n)\right]\right\},
$$

with $p_{F}^{(j)}(n)=\sqrt{\tilde{\mu}_{j}-M_{j}^{\star(0)^{2}}-2 e B n}$. From Eq. (49), the latter equation, and using the property of the Bessel function, $J_{m}(b=$ $0)=\delta_{m 0}$, we find:

$$
S_{j}\left[\frac{J_{m}^{2}(0)}{E_{j}^{(0)^{k}}} D_{\|}^{(j)}\right]=\frac{1}{q} \frac{1}{2 \pi^{2}} \sum_{n=0}^{n_{\max }} e B \int_{0}^{\infty} d u \frac{e^{-u}}{E_{j n}^{(0) k+1}}(-1)^{n+1}\left(L_{n}(2 u)-L_{n-1}(2 u)\right)\left(\frac{p_{F}^{(j)}(n)}{\left(\frac{\omega}{q}\right)^{2}-\left(\frac{p_{F}^{(j)}(n)}{E_{j n}^{(0)}}\right)^{2}}\right),
$$


where $E_{j n}^{(0)}=\sqrt{M_{j}^{\star(0)}+p_{F}^{(j)^{2}}(n)+e B u}$. The explicit matrix elements of Eq. (54) are

$$
\begin{aligned}
& a_{11}=1+F^{p p} \mathrm{~L}_{p}^{(0)}, \quad a_{12}=F^{p n} \mathrm{~L}_{p}^{(0)}, \quad a_{13}=B_{A} \mathrm{~L}_{p}^{(0)}, \quad a_{14}=M^{\star(0)} B_{s} \mathrm{~L}_{p}^{(1)}, \quad a_{15}=M^{\star(0)} B_{s} \mathrm{~L}_{p}^{(1)} \\
& a_{21}=F^{n p} \mathrm{~L}_{n}^{(0)}, \quad a_{22}=1+F^{n n} \mathrm{~L}_{n}^{(0)}, \quad a_{23}=0, \quad a_{24}=M^{\star(0)} B_{s} \mathrm{~L}_{n}^{(1)}, \quad a_{25}=M^{\star(0)} B_{s} \mathrm{~L}_{n}^{(1)} \\
& a_{31}=B_{A} \mathrm{~L}_{e}^{(0)}, \quad a_{32}=0, \quad a_{33}=1-B_{A} \mathrm{~L}_{e}^{(0)}, \quad a_{34}=0, \quad a_{35}=0 \quad a_{41}=F^{p p} M^{\star(0)} \mathrm{L}_{p}^{(1)}, \quad a_{42}=F^{p n} M^{\star(0)} \mathrm{L}_{p}^{(1)}, \\
& a_{43}=B_{A} M^{\star(0)} \mathrm{L}_{p}^{(1)}, \quad a_{44}=1+B_{s} F^{p p} M^{\star(0)} \mathrm{L}_{p}^{(2)}, \quad a_{45}=B_{s} F^{p p} M^{\star(0)^{2}} \mathrm{~L}_{p}^{(2)} \\
& a_{51}=F^{n p} M^{\star(0)} \mathrm{L}_{p}^{(1)}, \quad a_{52}=F^{n n} M^{\star(0)} \mathrm{L}_{n}^{(1)}, \quad a_{53}=0, \quad a_{54}=B_{s} M^{\star(0)} \mathrm{L}_{n}^{(2)}, \quad a_{55}=1+B_{s} M^{\star(0)^{2}} \mathrm{~L}_{n}^{(2)},
\end{aligned}
$$

where

$$
F^{i j}=-\left(B_{v}+\tau_{i} \tau_{j} B_{\rho}+\frac{Q_{i} Q_{j}}{e^{2}} B_{A}\right)
$$

and

$$
\begin{aligned}
& B_{v}=\frac{1}{2 \pi^{2}} \frac{g_{v}^{2}}{\omega^{2}-\omega_{v}^{2}}\left(1-\frac{\omega^{2}}{q^{2}}\right) \\
& B_{\rho}=\frac{1}{2 \pi^{2}} \frac{\left(g_{\rho} / 2\right)^{2}}{\omega^{2}-\omega_{\rho}^{2}}\left(1-\frac{\omega^{2}}{q^{2}}\right) \\
& B_{s}=\frac{1}{2 \pi^{2}} \frac{g_{s}^{2}}{\omega^{2}-\omega_{s}^{2}}, \quad B_{A}=-\frac{e^{2}}{2 \pi^{2}} \frac{1}{q^{2}}
\end{aligned}
$$

with $\omega_{s}^{2}=\tilde{m}_{s}^{2}+\vec{q}^{2}, \omega_{v}^{2}=m_{v}^{2}+\vec{q}^{2}$, and $\omega_{\rho}^{2}=m_{\rho}^{2}+\vec{q}^{2}$. The generalized Lindhard functions for the proton and electron are defined as:

$$
\mathrm{L}_{j}^{(k)}=e B \sum_{n=0}^{n_{\max }} \int_{0}^{\infty} d u\left[L_{n}(2 u)-L_{n-1}(2 u)\right] \frac{e^{-u}}{E_{j n}^{(0) k+1}}(-1)^{n+1}\left(\frac{p_{F}^{(j)}(n)}{\left(\frac{\omega}{q}\right)^{2}-\left(\frac{p_{F}^{(j)}(n)}{E_{j n}^{(0)}}\right)^{2}}\right)
$$

Notice that we have defined the generalized Lindhard function, $\mathrm{L}_{j}^{(k)}$, such that the present Vlasov magnetized dispersion relation and the corresponding nonmagnetized Vlasov dispersion relation of Ref. [25] differs only in what concerns this function. Hence, the limit $B=0$ is easily obtained.

\section{APPENDIX C: DISPERSION RELATION MATRIX ELEMENTS FOR TRANSVERSE MODES}

An equation similar to Eq. (B1) provides the necessary solutions for the various $S_{j}$ functions. However, an important complication arises in the context of the transverse modes, in that the argument of the Bessel functions is not zero. This means one needs to compute the contribution of the Bessel functions and perform the integrals:

$$
S_{j}\left[\frac{J_{m}^{2}(b)}{E_{j}^{(0)^{k}}} \frac{m}{b} D_{\perp}^{(j)}\right]=\frac{1}{2 \pi^{2}} \sum_{m=-\infty}^{\infty} \int_{-\infty}^{\infty} d p_{\|} \int_{0}^{\infty} d p_{\perp} p_{\perp} \frac{m}{b} \frac{J_{m}^{2}(b)}{E_{j}^{\star(0) k}} \frac{D_{\perp}^{(j)}}{\omega+\frac{Q_{j} B m}{E_{j}^{(0) k}}} .
$$

Next, we obtain $S_{j}$ by taking the derivative of the equilibrium distribution function, Eq. (21),

$$
D_{\perp}^{(j)}=\frac{\partial}{\partial p_{\perp}} f^{(0)(j)}=\sum_{n=0}^{\infty}\left[L_{n}\left(2 w^{2}\right)+L_{n-1}\left(2 w^{2}\right)\right](-1)^{n+1} e^{-w^{2}} \frac{2 p_{\perp}}{e B} \theta\left(p_{F}^{(j)}(n)-p_{\|}\right),
$$

and substituting the latter result in Eq. (C1). After some simple manipulations, by separating negative and positive (as well as zero) values of $m$, and using properties of the Bessel functions in order to reduce the integrals to more usual forms, one obtains:

$$
\begin{aligned}
S_{j}\left[\frac{J_{m}^{2}(b)}{E_{j}^{(0)^{k}}} \frac{m}{b} D_{\perp}^{(j)}\right]= & \frac{1}{2 \pi^{2}} \frac{1}{q} \sum_{n=0}^{n_{\max }} \int_{0}^{p_{F}^{(j)}(n)} d p_{\|} 8(e B) \int_{0}^{\infty} d p_{\perp} p_{\perp}\left[L_{n}\left(2 w^{2}\right)+L_{n-1}\left(2 w^{2}\right)\right](-1)^{n+1} e^{-w^{2}} \\
& \times \frac{1}{E_{j}^{\star(0)^{k+1}}} \sum_{m=0}^{\infty}\left(1-\frac{\delta_{m 0}}{2}\right) \frac{m^{2} J_{m}(b)^{2}}{\omega^{2}-\frac{\left(Q_{j} B m\right)^{2}}{E_{j}^{\star(0)^{2}}}} \equiv \frac{1}{q} \frac{1}{2 \pi^{2}} L_{j}^{(k)} .
\end{aligned}
$$


These equations simplify considerably in some particular cases of interest. For instance, to determine the spinodal boundary, we impose $\omega=0$. Below, we show the results for $L_{j}^{(k)}$ in this particular case:

$$
L_{j}^{(k)}=\frac{8}{e B} \int_{0}^{\infty} d p_{\perp} p_{\perp} \frac{(-1)^{n} e^{-w^{2}}}{E_{j}^{\star(0)}} \sum_{n=0}^{n_{\max }} \int_{0}^{p_{F}^{(j)}(n)} d p_{\|}\left[L_{n}(2 u)+L_{n-1}(2 u)\right] \sum_{m=0}^{\infty}\left(1-\frac{\delta_{m 0}}{2}\right) J_{m}^{2}(b) .
$$

The latter equation can be simplified further by using the following Bessel function property [23]:

$$
\sum_{m=0}^{\infty}\left(1-\frac{\delta_{m 0}}{2}\right) J_{m}^{2}(b)=\frac{1}{2}
$$

The special cases involved in the calculation of the dispersion relations are the following:

$$
\begin{aligned}
L_{j}^{(0)}= & \frac{2}{e B} \sum_{n=0}^{n_{\max }} \int_{0}^{\infty} d p_{\perp} p_{\perp}\left[L_{n}\left(2 w^{2}\right)+L_{n-1}\left(2 w^{2}\right)\right](-1)^{n} e^{-w^{2}} \\
& \times\left[p_{F}^{(j)}(n) \sqrt{M_{j}^{\star(0)^{2}}+p_{\perp}^{2}+p_{F}^{(j)}(n)^{2}}++\left(M_{j}^{\star(0)^{2}}+p_{\perp}^{2}\right) \ln \frac{p_{F}^{(j)}(n)+\sqrt{M_{j}^{\star(0)^{2}}+p_{\perp}^{2}+p_{F}^{(j)}(n)^{2}}}{\sqrt{M_{j}^{\star(0)^{2}}+p_{\perp}^{2}}}\right] \\
L_{j}^{(1)}= & \frac{4}{e B} \sum_{n=0}^{n_{\max }} \int_{0}^{\infty} d p_{\perp} p_{\perp}\left[L_{n}\left(2 w^{2}\right)+L_{n-1}\left(2 w^{2}\right)\right](-1)^{n} e^{-w^{2}} p_{F}^{(j)}(n) \\
L_{j}^{(2)}= & \frac{4}{e B} \sum_{n=0}^{n_{\max }} \int_{0}^{\infty} d p_{\perp} p_{\perp}\left[L_{n}\left(2 w^{2}\right)+L_{n-1}\left(2 w^{2}\right)\right](-1)^{n} e^{-w^{2}} \ln \frac{p_{F}^{(j)}(n)+\sqrt{M_{j}^{\star(0))^{2}}+p_{\perp}^{2}+p_{F}^{(j)}(n)^{2}}}{\sqrt{M_{j}^{\star(0)^{2}}+p_{\perp}^{2}}} .
\end{aligned}
$$

We have chosen the notation $L_{j}^{k}$ to mirror the generalized Lindhard functions, given in Appendix B, and, in fact, substituting the equations above for those functions, one ends with a matrix for the transverse modes that is identical to the one obtained for the longitudinal modes, (54).

[1] R. C. Duncan and C. Thompson, Astrophys. J. 392, L9 (1992); C. Thompson and R. C. Duncan, Mon. Not. R. Astron. Soc. 275, 255 (1995).

[2] SGR/APX online catalog, http://www.physics.mcgill.ca/ pulsar/magnetar/main.html

[3] J. A. Pons, D. Viganò, and N. Rea, Nat. Phys. 9, 431 (2013).

[4] D. G. Ravenhall, C. J. Pethick, and J. R. Wilson, Phys. Rev. Lett. 50, 2066 (1983); C. J. Horowitz, M. A. Pérez-García, and J. Piekarewicz, Phys. Rev. C 69, 045804 (2004); H. Sonoda, G. Watanabe, K. Sato, K. Yasuoka, and T. Ebisuzaki, ibid. 77, 035806 (2008); A. S. Schneider, D. K. Berry, C. M. Briggs, M. E. Caplan, and C. J. Horowitz, ibid. 90, 055805 (2014).

[5] J. Fang, H. Pais, S. Avancini, and C. Providência, Phys. Rev. C 94, 062801(R) (2016).

[6] J. Fang, H. Pais, S. Pratapsi, S. Avancini, J. Li, and C. Providência, Phys. Rev. C 95, 045802 (2017).

[7] J. Fang, H. Pais, S. Pratapsi, and C. Providência, Phys. Rev. C 95, 062801(R) (2017).

[8] C. Providência, L. Brito, S. S. Avancini, D. P. Menezes, and Ph. Chomaz, Phys. Rev. C 73, 025805 (2006).

[9] G. A. Lalazissis, J. König, and P. Ring, Phys. Rev. C 55, 540 (1997).

[10] B. G. Todd-Rutel and J. Piekarewicz, Phys. Rev. Lett. 95, 122501 (2005).

[11] D. Vasak, M. Gyulassy, and H.-Th. Elze, Ann. Phys. (NY) 173, 462 (1987).

[12] P. Zhuang and U. Heinz, Ann. Phys. (NY) 245, 311 (1996).
[13] K. Weber et al., Nucl. Phys. A 539, 713 (1992).

[14] B. Blattel, V. Koch, and U. Mosel, Rep. Prog. Phys. 56, 1 (1993).

[15] H.-Th. Elze, M. Gyulassy, and V. Vasak, Nucl. Phys. B 276, 706 (1986).

[16] R. D. Tenreiro and R. Hakim, Phys. Rev. D 15, 1435 (1977).

[17] R. D. Tenreiro and R. Hakim, J. Phys. A 10, 1525 (1977).

[18] R. Hakim and J. Heyvaerts, Phys. Rev. A 18, 1250 (1978).

[19] R. Hakim and H. Sikak, Ann. Phys. 139, 230 (1982).

[20] M. Nielsen and J. da Providência, Phys. Rev. C 40, 2377 (1989).

[21] M. Nielsen, C. Providência, and J. da Providência, Phys. Rev. C 44, 209 (1991).

[22] M. H. Johnson and B. A. Lippmann, Phys. Rev. 76, 828 (1949).

[23] I. S. Gradshteyn and I. M. Ryzhik, Table of Integrals, Series and Products, 7th ed. (Academic Press, New York, 2007).

[24] A. Broderick, M. Prakash, and J. M. Lattimer, Astrophys. J. 537, 351 (2000).

[25] S. S. Avancini, L. Brito, D. P. Menezes, and C. Providência, Phys. Rev. C 71, 044323 (2005).

[26] C. Oberman and A. Ron, Phys. Rev. 130, 1291 (1963).

[27] D. C. Kelly, Phys. Rev. 134, A641 (1964).

[28] K. Kiuchi and S. Yoshida, Phys. Rev. D 78, 044045 (2008).

[29] J. Frieben and L. Rezzolla, Mon. Not. R. Astron. Soc. 427, 3406 (2012).

[30] Y. J. Chen, Phys. Rev. C 95, 035807 (2017). 
[31] A. Rabhi, C. Providência, and J. da Providência, Phys. Rev. C 79, 015804 (2009).

[32] L. Brito, C. Providência, A. M. Santos, S. S. Avancini, D. P. Menezes, and Ph. Chomaz, Phys. Rev. C 74, 045801 (2006).

[33] S. S. Avancini, D. P. Menezes, M. D. Alloy, J. R. Marinelli, M. M. W. Moraes, and C. Providência, Phys. Rev. C 78, 015802 (2008).

[34] H. Pais, A. Santos, L. Brito, and C. Providência, Phys. Rev. C 82, 025801 (2010).
[35] F. Grill, C. Providência, and S. S. Avancini, Phys. Rev. C 85, 055808 (2012).

[36] S. K. Lander, Astrophys. J. Lett. 824, L21 (2016).

[37] B. Haskell, Int. J. Mod. Phys. E 24, 1541007 (2015).

[38] A. I. Chugunov, M. E. Gusakov, and E. M. Kantor, MNRAS 468, 291 (2017).

[39] A. Firouzi, D. J. Schaefer, S. H. Tolbert, G. D. Stucky, and B. F. Chmelka, Am. Chem. Soc. 119, 9466 (1997); Y. Dou, S. Dong, and J. Hao, Langmuir 30, 1266 (2014). 